\title{
Dynamically-Sampled Bivariate Empirical Mode Decomposition
}

\author{
Naveed ur Rehman, Muhammad Waqas Safdar, Ubaid ur Rehman, and Danilo P. Mandic
}

\begin{abstract}
A novel scheme for selecting projection vectors in bivariate empirical mode decomposition (BEMD) is proposed in order to enable accurate signal decomposition at lower computational complexity. Unlike existing algorithms which use a static uniform scheme for the distribution of projection vectors, the proposed scheme examines local curvature in multidimensional spaces to produce a data-adaptive set of direction vectors for taking signal projections. This is achieved by aligning the density of projection vectors according to the empirical distributions of angles where the signal exhibits highest local dynamics. We show that the proposed methodology outperforms the existing schemes for a small number of signal projections. The proposed algorithm is verified via illustrative simulations demonstrating accurate local mean estimation and mode extraction.
\end{abstract}

Index Terms-Bivariate EMD, inverse transform sampling, Menger curvature.

\section{INTRODUCTION}

$\mathbf{T}$ HE empirical mode decomposition (EMD) [1] is an established data-driven method for multiscale analysis of nonlinear and nonstationary time series. It represents an arbitrary signal $x(t)$ via a sum of its inherent oscillatory modes $c_{m}(t)$, termed intrinsic mode functions (IMFs), and a residual $r(t)$ as

$$
x(t)=\sum_{m=1}^{M} c_{m}(t)+r(t)
$$

The IMFs $c_{m}(t)$ are zero-mean AM/FM modulated components which lend themselves to highly localized time-frequency (T-F) estimates by combining EMD and the Hilbert transform (HT)-the so called Hilbert-Huang spectrum. Another advantage is that the EMD makes no a priori assumptions regarding input data, making the algorithm popular in a wide range of applications.

Recent developments in sensor and data acquisition technologies have enabled routine recordings of multivariate signals, yet algorithms which can cater for nonlinear and nonstationary multivariate data are scarce. In the EMD context, for bivariate data

Manuscript received January 03, 2014; revised April 07, 2014; accepted April 07, 2014. Date of publication April 16, 2014; date of current version April 28, 2014. The associate editor coordinating the review of this manuscript and approving it for publication was Prof. Gitta Kutyniok.

N. Rehman and M. W. Safdar are with the Department of Electrical Engineering, COMSATS Institute of Information Technology, Islamabad, Pakistan (e-mail: nrehman.comsats@gmail.com; vaqas_001@yahoo.com).

U. Rehman is with Electrical Engineering and Computer Science Department, University of Kassel, 34109 Kassel, Germany (e-mail: ubaid515@yahoo.com).

D. Mandic is with the Department of Electrical Engineering, Imperial College London, London SW7 2AZ U.K (e-mail: d.mandic@imperial.ac.uk).

Color versions of one or more of the figures in this paper are available online at http://ieeexplore.ieee.org.

Digital Object Identifier 10.1109/LSP.2014.2317773 (containing two data channels) these include: i) Complex EMD (CEMD) [2]; ii) Rotation-invariant EMD (RI-EMD) [3]; and iii) Bivariate EMD (BEMD) [4]. The computation of the local mean is the most important and challenging step in developing such methods since the complex field $\mathbb{C}$ is not ordered and the relations " $<$ " and " $>$ " have no meaning. To this end, RI-EMD and BEMD take projections of a bivariate signal in multiple directions, and subsequently calculate signal envelopes by interpolating the extrema of those projections. Finally, the local mean is computed by averaging these multiple univariate envelopes. Owing to its fully complex nature, both RI-EMD and BEMD produce equal number of IMFs for real and imaginary components, however, they differ in the number of projections they take to calculate the local mean: while RI-EMD takes only two projections, BEMD can employ any number of projections in $2 \mathrm{D}$ and is thus better suited to handle fast-changing signal dynamics. More recently, a fully multivariate [5] extension of EMD has been proposed, which extends the BEMD principle to multivariate signals containing any number of data channels.

The BEMD and MEMD algorithms have already been proven in a wide range of applications, ranging from environmental condition monitoring to brain-computer interface (BCI). However, they employ static distributions of direction vectors for taking signal projections in multidimensional spaces, not considering any information regarding the changing dynamics of the signal at hand. While their global sampling strategy produces accurate results for a large number of projections, in cases where computational complexity is an issue, sampling strategies should be local and data dependent. To address this issue, a nonuniform sampling scheme for the BEMD was recently introduced which employs non-circular statistics of a complex signal to identify the principal directions for taking signal projections [6]. Albeit an improvement over uniform sampling strategies, this approach considers global information about the correlation and power of individual data channels, and is thus best suited for stationary data, hence limiting its usefulness for real world applications.

To resolve this issue, we propose here a novel scheme to determine the direction vectors for taking signal projections based on local signal dynamics. This is a local scheme which assigns the distribution of direction vectors according to the angles where the signal exhibits maximum variations. We show that this not only produces more accurate estimates of the local mean but also results in significant savings in computational complexity, as fewer projections are required to obtain the local mean. The proposed method operates by first estimating an empirical cumulative distribution function (CDF) of direction vectors (angles) based on the local signal curvature; subsequently, samples are drawn from the estimated empirical CDF using the inverse transform sampling (ITS) method, yielding a 
set of angles where maximum signal variations are exhibited. The proposed method is shown to outperform standard bivariate EMD via simulations on both synthetic and real world signals.

\section{BIVARIATE EMD (BEMD)}

The BEMD algorithm [4] extends the notion of 'oscillations' in standard EMD to 'rotations' in 2D and attempts to decompose a bivariate signal in terms of slower and faster rotations through a process referred to as the sifting algorithm. It takes multiple projections $\left\{w_{\theta_{v}}(t)\right\}_{v=1}^{V}$ of input signal along uniform angle set and computes corresponding bivariate envelope curves $\left\{e_{\theta_{v}}(t)\right\}_{v=1}^{V}$ by interpolating between the maxima points of the projections; subsequently a nonlinear elementary operator $\mathbb{S}$ is applied on the signal until some stopping criterion is met, yielding multiple bivariate components in the process. Algorithm 1 lists the steps involved in the BEMD algorithm.

\section{Algorithm 1 The sifting process in BEMD}

1: Given a bivariate signal $z(t)$, obtain $V$ signal projections, $\left\{w_{\theta_{v}}(t)\right\}_{v=1}^{V}$, along uniform directions $\theta_{v}$ via

$$
w_{\theta_{v}}(t)=\mathcal{R}\left(e^{-i \theta_{v}} z(t)\right)
$$

where $\mathcal{R}(\cdot)$ denotes the real part of a complex number, and $\theta_{v}=2 v \pi / V$ for $v=1, \ldots, V$;

2: Find the locations $\left\{t_{j}^{v}\right\}_{v=1}^{V}$ corresponding to the maxima of $\left\{w_{\theta_{v}}(t)\right\}_{v=1}^{V}$;

3: Interpolate (using spline interpolation) between the maxima points $\left[t_{j}^{v}, z\left(t_{j}^{v}\right)\right]$ component-wise, to obtain the bivariate (complex-valued) envelope curves $\left\{e_{\theta_{v}}\right\}_{v=1}^{V}$;

4: $\quad$ Calculate the bivariate mean, $m_{2}(t)$, of all the envelope curves

$$
m_{2}(t) \approx \frac{1}{V} \sum_{v=1}^{V} e_{\theta_{v}}(t)
$$

5: $\quad$ Subtract the mean $m(t)$ to give $\mathbb{S}[z](t)=z(t)-m(t)$.

\section{DyNAMICALlY-SAMPLED BEMD (DS-BEMD)}

The rationale behind the proposed DS-BEMD method is to incorporate the information regarding local signal dynamics into BEMD by generating a set of angles (directions) where the input signal exhibits maximum variation. The aim is to enable accurate local mean estimation for a smaller number of projection vectors $V$, increasing the overall efficiency of the method. The proposed method employs: 1) Menger curvature measure to model local signal dynamics resulting in the distribution of angles (directions) exhibiting high-dynamics, and 2) Inverse Transform Sampling (ITS) to generate angles from that distribution.

\section{A. Estimating Local Dynamics via Menger Curvature}

The curvature of a function measures its local deviation from a straight line, and is a natural choice to quantify local signal dynamics, since intuitively, the larger the deviation from straight line, the higher the dynamics (variations) of a signal (function). A convenient means of estimating curvature for real world sig-

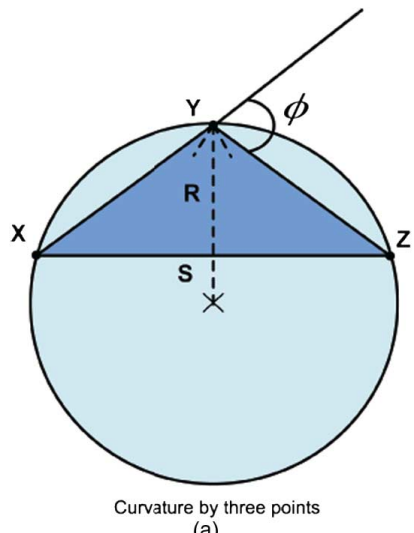

(a)

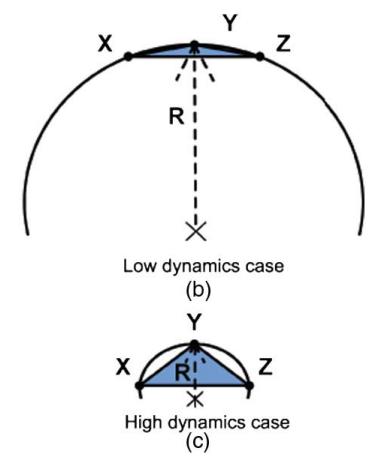

Fig. 1. Computation of Menger curvature (a) For three points $X, Y$, and $Z$ on a curve, the curvature is calculated through curvature radius $R$ (eq. (4)), the triangle $X Y Z$ (eq. (5)), and the angle $\phi$ (eq. (6)). (b) Low dynamics case corresponding to high curvature radius $R$. (c) High dynamics case corresponding to low curvature radius $R$.

nals is through the Menger Curvature, which defines the curvature radius $R$ as the limit of the radius of a circle containing three points of a curve, as shown in Fig. 1(a). The radius $R$ of the osculating circle, defined as a circle at a point $Y$ on a curve $C$ which passes through the point $Y$ and some infinitesimally close points to $Y$ on $C$, is inversely proportional to the curvature $\kappa$ of the curve at the middle point $Y$, given by:

$$
\kappa(X, Y, Z)=\frac{1}{R}
$$

Considering a triangle $X Y Z$ passing through the three points $(X, Y, Z)$ of a curve, the side lengths $|X Y|,|Y Z|$, and $|Z X|$ and the area $A$ of the triangle are related as:

$$
\kappa(X, Y, Z)=1 / R=\frac{4 A}{|X Y||Y Z||Z X|}
$$

Another way of computing the Menger curvature is through the identity:

$$
\kappa(X, Y, Z)=\frac{2 \sin (\phi)}{S}
$$

where $\phi$ denotes the angle corresponding to the Y-corner of the triangle and $S=|Z-X|$ is the length of the hypotenuse, as shown in Fig. 1(a). From eq. (4) and Fig. 1, observe that an osculating circle with a higher value of $R$ corresponds to lower curvature and slower dynamics (Fig. 1(b)), whereas a circle with a smaller value of $R$ corresponds to higher curvature and faster dynamics (Fig. 1(c)).

In the proposed method, the Menger curvature measure is computed via eq. (5) to quantify local signal dynamics of an input bivariate signal $z(k)=x(k)+i y(k)$ at each point $\{x(k), y(k)\}$. Subsequently, $L$ points exhibiting high dynamics are selected and their corresponding angles $\left\{\theta_{l}\right\}_{l=1}^{L}$ are calculated via eq. (2). The empirical cumulative distribution, $F\left(\theta_{l}\right)$, of those angles is then estimated which is used a reference to compute the angles exhibiting high dynamics via the ITS method [7], as demonstrated next.

\section{B. Selecting Angle Set via Inverse Transform Sampling}

The ITS is a popular method to produce random variates with an arbitrary distribution and we shall employ it to generate de- 
terministic angle set $\theta_{\kappa}$ based on the distribution $F\left(\theta_{l}\right)$ of signal angles exhibiting high dynamics. It operates on $F\left(\theta_{l}\right)$ by: i) generating a set $U$ of $V$ uniformly sampled points in the range [0,1]; ii) finding the smallest integer $I$ such that $U \leq F\left(\theta_{I}\right)$; and iii) returning the desired angle set as $\left\{\theta_{\kappa}^{v}\right\}_{v=1}^{V}=\theta_{I}$. This way, we obtain $V$ angles exhibiting maximum signal variation. For very small values of $V$, the uniform samples $U$ may not cover the whole range of $[0,1]$ adequately and hence the proposed method selects the first $V$ angles corresponding to the maximum values from the histogram of $\theta_{l}$. The steps involved in the computation of the angle set $\theta_{\kappa}^{v}$ in DS-BEMD are outlined in Algorithm 2. The angle set $\theta_{\kappa}^{v}$ then replaces the uniform angle set $\theta_{v}$ (eq. (2)) used in the standard BEMD in step 1; the rest of the operations of the DS-BEMD, including the computation of local mean, are the same as those in standard BEMD (steps 2-5 in Algorithm 1).

\section{Algorithm 2 Dynamically-Sampled BEMD (DS-BEMD)}

1: Estimate the curvature $\kappa$ of an input bivariate/complex signal $z(k)=x(k)+i y(k)$, with $x$ and $y$ being sampled at the same rate and each of length $N$, using eq. (5);

2: $\quad$ Select first $L$ points exhibiting top curvature values;

3: Find $\mathrm{L}$ angles $\left\{\theta_{l}\right\}_{l=1}^{L}$ corresponding to the selected data points exhibiting high dynamics via eq. (2);

\section{4: $\quad$ if $V<=6$ then}

5: $\quad$ Generate histogram $h_{\theta_{l}}$ with $H$ number of bins of the resulting angles $\theta_{l}$;

6: $\quad$ Select the first $V \leq H$ angles, $\left\{\theta_{\kappa}^{v}\right\}_{v=1}^{V}$ corresponding to the peak values of $h_{\theta_{l}}$;

\section{7: $\quad$ else}

8: $\quad$ Find the CDF $F\left(\theta_{l}\right)$ of the resulting angles $\theta_{l}$;

9: $\quad$ Generate $V$ deterministic uniform samples $U$ in the range $[0,1]$;

10: $\quad$ Find the smallest integer $I$ such that $U \leq F\left(\theta_{I}\right)$;

11: $\quad$ Choose $\theta_{\kappa}^{v}=\theta_{I}$ where $v=1, \ldots, V$.

\section{2: $\quad$ end if}

Fig. 2 illustrates the operation of DS-BEMD for both balanced circular white Gaussian noise (WGN) and a non-circular synthetic combination of sinusoids. The scatter plot of circular WGN data is shown in Fig. 2(a) and has equal powers in both data channels resulting in an approximately uniform histogram and a linear CDF, as shown in Figs. 2(c) and (e) respectively. The DS-BEMD, therefore, yielded uniformly distributed direction vectors along a circle, as shown in Fig. 2(g).

The operation of DS-BEMD on a benchmark non-circular bivariate signal, $s_{31}$ used in [8], is also shown in Fig. 2(b) and Fig. 2(d), which show respectively the original signal and the histogram of its angles according to data dynamics, together with the kernel smoothing estimate of the histogram (dashed line). Observe that the histogram of direction vectors now has peaks at $\theta_{1}=90^{\circ}$ and $\theta_{2}=270^{\circ}$, resulting in the corresponding empirical CDF exhibiting 'jumps' at those angles, as shown in

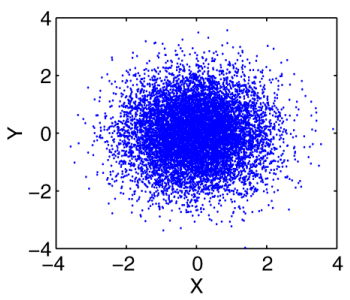

(a)

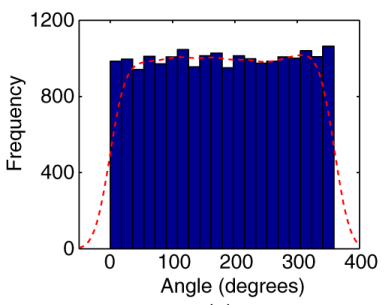

(c)

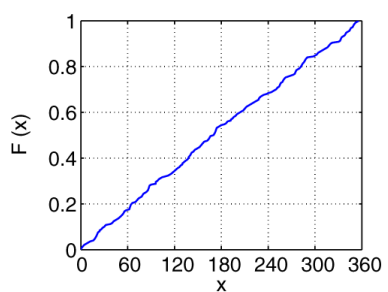

(e)

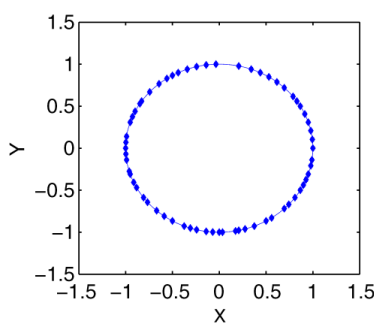

(g)

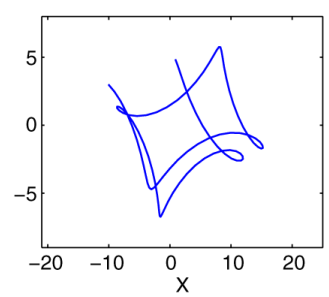

(b)

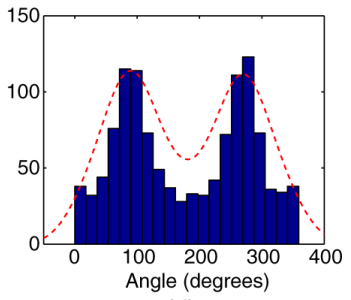

(d)

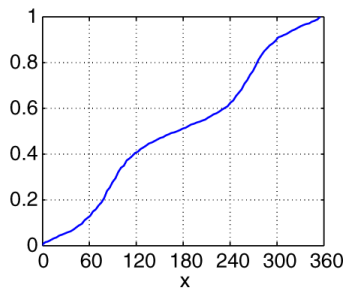

(f)

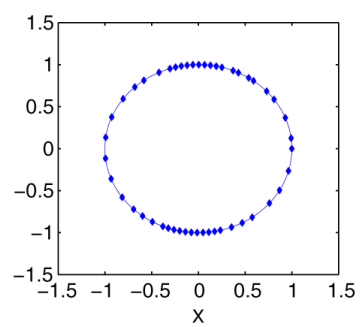

(h)
Fig. 2. $V=48$ projection vectors obtained from the DS-BEMD for circular white Gaussian noise (WGN) and synthetic non-circular bivariate data. The corresponding histograms and CDFs are given respectively in (c-d) and (e-f).

Fig. 2(f). For an optimised and fully data driven BEMD operation, the distribution of projection angles should therefore be denser along the angles $\theta_{1}=90^{\circ}$ and $\theta_{2}=270^{\circ}$, which is achieved by the proposed method as demonstrated in Fig. 2(h).

\section{Computational Aspects of DS-BEMD}

Increasing the number of projections $V$ improves the accuracy of local mean estimation in BEMD, but at the same time it significantly increases the computational complexity of the algorithm. This can be observed from the following relation which gives the numerical complexity of BEMD [8] as a function of the input signal length $N$, number of projections $V$, total number of IMFs $M$, and the number of detected extrema $M_{k}\left(d_{m, k}, v\right)$ in the $v$ th projection of $m$ th IMF and $k$ th iteration:

$$
C=\sum_{m=1}^{M} \sum_{k=1}^{K_{m}} N(11 V+2)+\sum_{m=1}^{M} \sum_{k=1}^{K_{m}} \sum_{v=1}^{V} 15 M_{k}\left(d_{m, k}, v\right)
$$

The proposed DS-BEMD reduces the complexity of BEMD by significantly decreasing the number of projections $V$ for the same accuracy, owing to its projection scheme which matches signal dynamics. The added computational cost of DS-BEMD includes: a) computation of Menger curvature of order $\mathcal{O}(N)$; 


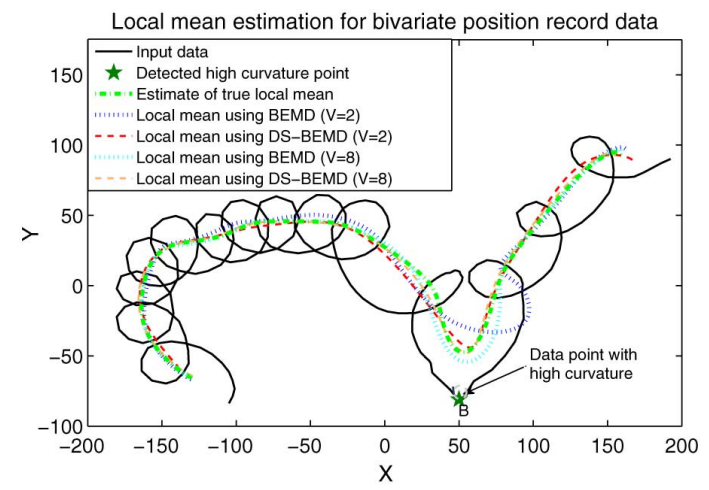

Fig. 3. Local mean estimates for real world bivariate data obtained from standard BEMD method (dotted lines) and the proposed method (dashed lines) using $V=2$ and $V=8$ projection vectors. The reference local mean estimate (dashed-dotted line) is also shown.

TABLE I

Pairwise Euclidean Distance from Reference Local Mean

\begin{tabular}{l|l|l|l|l|l|l|l}
\hline No. of Projections $V$ & 2 & 4 & 8 & 16 & 24 & 32 & 128 \\
\hline$P D$ (BEMD) & 26.7 & 11.6 & 10.0 & 2.6 & 2.1 & 2.0 & 0.32 \\
\hline$P D$ (DS-EMD) & 10.1 & 5.6 & 6.2 & 2.4 & 0.83 & 0.68 & 0.34 \\
\hline
\end{tabular}

b) estimation of cumulative distribution function $F(\theta)$ of order $\mathcal{O}(L \log L)$, where $L \ll N$; and c) ITS computation of order $\mathcal{O}(V \log V)$, where $V \ll N$. The benefits of reducing the value of $V$ in eq. (7) typically outweigh the added computational costs of DS-BEMD, making it more cost effective and accurate as compared to the standard BEMD.

\section{Simulation Results}

We next illustrate the advantages of the proposed DS-BEMD method on both real world and synthetic data.

\section{A. Local Mean Estimation}

In the first set of simulations, we analysed a real world position record data ${ }^{1}$ from a buoyant subsurface float used to track the motion of dense salty water from the Mediterranean sea during the "Eastern Basin" experiment [9]. Fig. 3 shows the local mean estimates obtained from standard BEMD (dotted lines) and the proposed DS-BEMD (dashed lines) by using $V=$ 2 and $V=8$ projection vectors for a single sifting iteration. The plots of the original data (black thick line) together with the estimates of true local mean or ground truth (green dashed-dotted line) are also shown. The true local mean was estimated by taking a very large number of direction vectors $(V=1024)$ in standard BEMD. It can be noticed that while DS-BEMD provided a more accurate estimate of the local mean at point $B$ exhibiting high dynamics, the local mean obtained from standard BEMD for $V=2$ went even outside of the signal boundaries at the location $[X=100, Y=-25]$, highlighting the need for data-dependent local mean estimation.

Table I quantifies the accuracy of the local mean estimates obtained from BEMD and DS-BEMD for a range of values of $V$. The sum of the standardized Euclidean distance, termed $P D$, between pairs of observations corresponding to the reference local mean and those obtained from the standard and proposed methods was used as the performance metric. The lower the value of $P D$, the smaller the deviation from the true local mean.

\footnotetext{
${ }^{1}$ We added a few data points synthetically in order to introduce some sharp dynamics in the input data at around $[X=50, Y=-80]$.
}

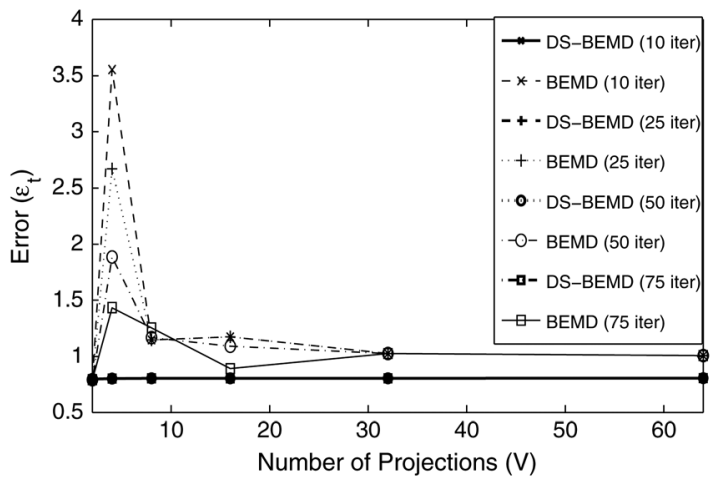

Fig. 4. Mode extraction error (given in eq. (8)) plots of dynamically sampled vs standard BEMD algorithm for a range of $V$ and $S$.

Observe that the proposed method produced much more accurate estimates of the local mean, especially for small to medium number of projection vectors $V$. For large values of $V$, both methods converged to the true local mean, as indicated by very small values of $P D$ in those cases.

\section{B. Intrinsic Mode Extraction}

We next evaluated the performance of DS-BEMD against the standard BEMD method in terms of the accuracy in signal extraction. The input bivariate data was a synthetic data set $s 21$ used in [8]. The performance criterion was an error signal $\epsilon_{t}$, a sum of individual component errors, given by:

$$
\epsilon_{t}=\sum_{m=1}^{M} \frac{\sum_{k=1}^{N}\left|c_{m}(k)-\hat{c}_{m}(k)\right|^{2}}{\sum_{k=1}^{N}\left|c_{m}(k)\right|^{2}}
$$

where $c_{m}(k)$ and $\hat{c}_{m}(k)$ denote respectively the desired (ground truth) and extracted IMF. Fig. 4 illustrates the mode extraction errors for the standard and the DS-BEMD methods, produced by varying both the number of projections $V$ and the sifting iterations $S$. Observe that the proposed method performed significantly better than the standard BEMD over the whole range of values of $V$ and $S$; in fact, the proposed method converged for a very small number of projection vectors $V=2$ and sifting iterations $S=10$. On the other hand, the standard BEMD exhibited significant error for small to moderate values of $V$ and $S$, mainly due to the mode mixing problem. The improvement in standard BEMD for higher $V$ was expected, since for a large number of direction vectors the static distribution of projections is also capable of tracking data dynamics, yielding smaller errors.

\section{CONCLUSIONS}

We have introduced a novel algorithm for selecting projection vectors within the bivariate EMD method. This has been achieved by incorporating information from local signal dynamics so as to yield enhanced estimation of the local mean, a key step in EMD algorithms. The proposed method is local and employs the curvature in multidimensional spaces to generate an empirical nonuniform distribution of angles corresponding to the dynamics of input data, whereby projection vectors (angles) in directions of highest curvature are selected for local mean estimation by using the inverse transform sampling (ITS). The performance advantage of DS-BEMD over standard BEMD has been shown to be significant for a low number of projections, a typical case in practical applications due to computational complexity constraints. 


\section{REFERENCES}

[1] N. E. Huang, Z. Shen, S. Long, M. Wu, H. Shih, Q. Zheng, N. Yen, C. Tung, and H. Liu, "The empirical mode decomposition and Hilbert spectrum for non-linear and non-stationary time series analysis," in Proc. Roy. Soc. A, 1998, vol. 454, pp. 903-995.

[2] T. Tanaka and D. P. Mandic, "Complex empirical mode decomposition,” IEEE Signal Process. Lett., vol. 14, no. 2, pp. 101-104, 2006.

[3] M. U. Altaf, T. Gautama, T. Tanaka, and D. P. Mandic, "Rotation invariant complex empirical mode decomposition," in Proc. IEEE Int. Conf. Acoustics, Speech, Signal Processing, 2007.

[4] G. Rilling, P. Flandrin, P. Goncalves, and J. M. Lilly, "Bivariate empirical mode decomposition," IEEE Signal Process. Lett., vol. 14, pp. 936-939, 2007.
[5] N. Rehman and D. P. Mandic, "Multivariate empirical mode decomposition," in Proc. Roy. Soc. A, 2010, vol. 466, no. 2117, pp. 1291-1302.

[6] A. Ahrabian, N. Rehman, and D. P. Mandic, "Bivariate empirical mode decomposition for unbalanced real-world signals," IEEE Signal Process. Lett., vol. 20, no. 3, pp. 245-248, 2013.

[7] L. Devroye, Non-Uniform Random Variate Generation. Berlin, Germany: Springer-Verlag, 1986.

[8] J. Fleureau, A. Kachenoura, L. Alberta, J. Nunes, and L. Senhadgi, "Multivariate empirical mode decomposition and application to multichannel filtering," Signal Process., vol. 91, pp. 9-12, 2011.

[9] P. Richardson, D. Walsh, L. Armi, M. Scroder, and J. F. Price, "Tracking three meddies with sofar floats," J. Phys. Oceanogr., vol. 19, pp. 371-383, 1989. 